\title{
Chemical evolution of bulges at high redshift
}

\author{
Antonio Pipino ${ }^{1,2}$, Francesca Matteucci ${ }^{2}$ and Annibale D'Ercole ${ }^{3}$ \\ ${ }^{1}$ Astrophysics, University of Oxford, Denys Wilkinson Building, Keble Road, \\ Oxford OX1 3RH, U.K. \\ email: axp@astro.ox.ac.uk \\ ${ }^{2}$ Dipartimento di Astronomia, Universita di Trieste, Via G.B. Tiepolo 11, 34100 Trieste, Italy \\ ${ }^{3}$ INAF-Osservatorio Astronomico di Bologna, Via Ranzani 1, 40127 Bologna, Italy
}

\begin{abstract}
We present a new class of hydrodynamical models for the formation of bulges (either massive elliptical galaxies or classical bulges in spirals) in which we implement detailed prescriptions for the chemical evolution of $\mathrm{H}, \mathrm{He}, \mathrm{O}$ and Fe. Our results hint toward an outside-in formation in the context of the supernovae-driven wind scenario. The build-up of the chemical properties of the stellar populations inhabiting the galactic core is very fast. Therefore we predict a non significant evolution of both the mass-metallicity and the mass- $[\alpha / \mathrm{Fe}]$ relations after the first 0.5 - 1 Gyr. In this framework we explain how the observed slopes, either positive or negative, in the radial gradient of the mean stellar $[\alpha / \mathrm{Fe}]$, and their apparent lack of any correlation with all the other observables, can arise as a consequence of the interplay between star formation and metal-enhanced internal gas flows.
\end{abstract}

Keywords. galaxies: elliptical and lenticular, cD; galaxies: abundances; Galaxy: bulge

\section{Introduction}

Negative metallicity radial gradients in the stellar populations are a common feature in spheroids (either elliptical and bulges, e.g. Carollo et al. 1993, Goudfroij et al. 1999 and Proctor et al. 2000, respectively). Moreover bulges remarkably follow many other fundamental constraints for ellipticals such as the mass-metallicity and the mass- $[\alpha / \mathrm{Fe}]$ relations, the only difference being that they might be rejuvenated systems (Thomas \& Davies 2006). To date, only a handful of observational works inferred the gradients in the $[\alpha / F e]$ ratios both elliptical galaxies (Melhert et al. 2003, Annibali et al. 2006, Sanchez-Blazquez et al. 2007) and galaxy bulges (Jablonka et al. 2007). These papers show that the slope in the $[\alpha / F e]$ gradient can be either negative or positive, with a mean value close to zero, and that it does not correlate with galactic properties (e.g. stellar mass for elliptical and Hubble type of the host spiral for bulges). To date such facts have not been properly addressed by any model of galaxy formation.

\section{The model}

We adopted a one-dimensional hydrodynamical model which follows the time evolution of the density of mass, momentum and internal energy of a galaxy, under the assumption of spherical symmetry. Our main novelty is that we follow the chemical evolution of several elements, namely $\mathrm{H}, \mathrm{He}, \mathrm{O}$ and Fe. This set of elements is good enough to characterize our simulated elliptical galaxy from the chemical evolution point of view. We refer the reader to Pipino, D'Ercole \& Matteucci (2007, PDM) for a comprehensive discussion of the adopted code and for the description of the models for massive spheroids. Concerning the galaxy bulges, instead, we assume that they are stellar systems with mass $\sim 2 \times 10^{10} M_{\odot}$ embedded in a $\sim 100$ times more massive Dark Matter halo. We neglect 
the presence of a disc, which requires a much longer timescale to be built. Several initial gas temperatures and density profiles have been tested. In this paper we show the results for fiducial model; this is the one, among all the models we ran, which gives the best match of the Milky Way bulge.

\section{Results and discussion}

All the models undergo an outside-in formation as suggested by Pipino, Matteucci \& Chiappini (2006, PMC), in the sense that star formation stops earlier in the outermost than in the innermost regions, owing to the onset of a galactic wind which proceeds from large radii (where the potential well is shallower) to the galactic core.

The mass-metallicity relation is satisfied, since our massive objects have an average metallicity in the stars which is supersolar (see PDM for details), whereas the simulated bulges have solar metallicity at most. At the same time we also satisfy the relation between $[\alpha / \mathrm{Fe}]$ and mass (see e.g. Thomas \& Davies 2006), being the central region of our massive spheroids more $\alpha$-enhanced that those of the bulges. These relations are in place after 0.5 - 1 Gyr since the beginning of the star formation. It is importan to underline that our findings regarding the build-up of both the mass-metallicity and the $[\alpha / \mathrm{Fe}]$-mass relations are real predictions and can be checked by on-coming observational campaigns.

\subsection{Massive ellipticals}

We find $[\mathrm{Fe} / \mathrm{H}]$ gradient slope in the range $-0.5--0.2$ dex per decade in radius and -0.3 dex per decade in radius for $[\mathrm{Z} / \mathrm{H}]$, in agreement with the observations (e.g. Annibali et al. 2007). Once transformed into predictions on the line-strenght indices, these gradients in the abundances lead to $d \mathrm{Mg}_{2} / \log \left(\mathrm{R}_{\text {eff }, *} / \mathrm{R}_{\text {core }, *}\right) \sim-0.06$ mag per decade in radius, again in agreement with the typical mean values measured for ellipticals by several authors and confirming the PMC best model predictions. The remarkable exception of some model with a steeper gradient seems to go in the direction of a few massive objects in the Ogando et al. (2005) sample.

By analysing typical massive ellipticals, we find that all the models that show values for their chemical properties, including the $[\mathrm{Fe} / \mathrm{H}]$ and the total metallicity gradients, within the observed ranges, show a variety of gradient in the $[\alpha / \mathrm{Fe}]$ ratio, either positive or negative, and one as no gradient at all. We find that the predicted variety of the gradients in the $[\alpha / \mathrm{Fe}]$ ratio can be explained by physical processes, generally not taken into account in simple chemical evolution models, such as metal-enhanced radial flows coupled with different initial conditions. Fig. 1 (left panel) gives a sketch of the various mechanisms at work (see PDM).

\subsection{Galaxy bulges}

Remarkably, all the results described in the previous section apply to smaller objects (but embedded in much more massive haloes) such as the galaxy bulges, although the gradient slopes are slightly smaller. Investigation of a possible dependence between slope and mass will be the subject of a forthcoming paper. Therefore our models suggest the gradient in the $[\alpha / \mathrm{Fe}]$ ratio to be related to the interplay between the velocity of the $\alpha$-enhanced radial flows, moving from the outer to the inner galactic regions, and the intensity and therefore duration of the SF formation process at any radius. In other words, if the flow velocity is fast relative to the star formation, the stars still forming at inner radii have time to form out of $\alpha$-enhanced gas coming from the outermost regions, thus flattening and even reversing the sign of the $[\alpha / \mathrm{Fe}]$ gradient (fig. 1, left panel). 

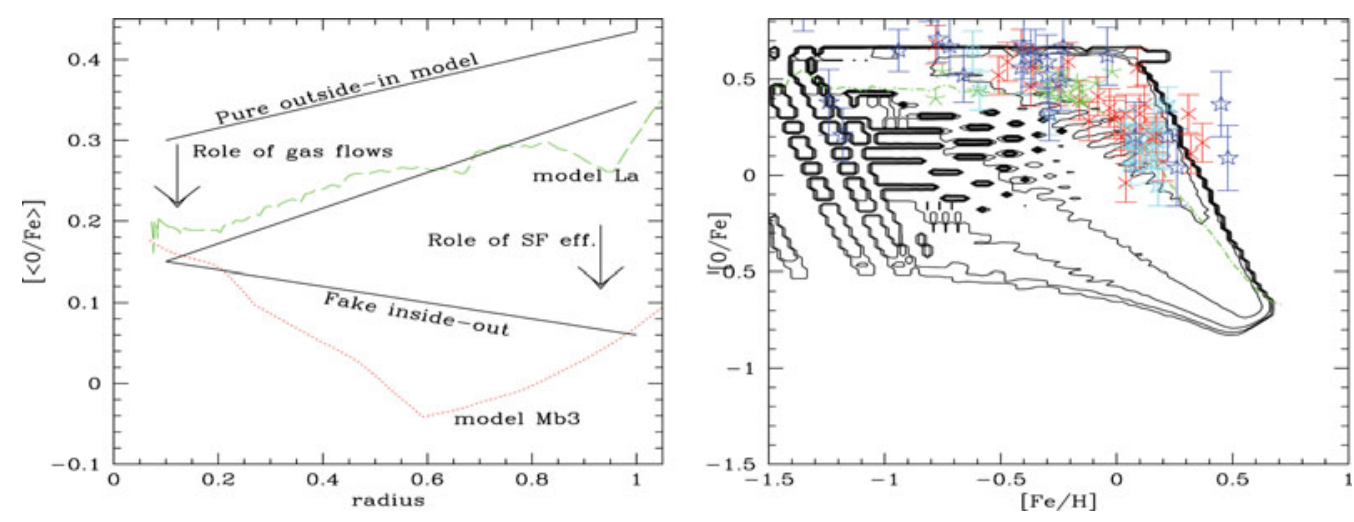

Figure 1. Left panel A sketch of the relative contribution of the gas flows strenght and the star formation efficiency $\nu$ to the creation of the final gradient for two particular cases from PDM. Pure outside-in model: hypothetical model with an outside-in formation, and $\nu$ constant with radius; fake inside-out model: hypothetical model with a strong variation of the star formation efficiency with radius. The abscissa is expressed in units of the effective radius. Right panel Contours: bidimensional metallicity distribution of stars as functions of $[\mathrm{Fe} / \mathrm{H}]$ and $[\mathrm{O} / \mathrm{Fe}]$ for the MW bulge. Dashed line: $[\mathrm{O} / \mathrm{Fe}]$ vs. $[\mathrm{Fe} / \mathrm{H}]$ in the gas of model (mass-weighted values on the gridpoints of each region). Symbols: recent data compiled by Ballero et al. (2007).

Moreover, the classical bulges allow us a further tool to calibrate our models. In fact, we can compare our model prediction to the properties of the resolved stellar population observed in the Milky Way bulge. In fig. 1 (right panel) we plot the distribution of stars formed out of gas with a given chemical pattern (i.e. a given $[\mathrm{Fe} / \mathrm{H}]$ and $[\mathrm{O} / \mathrm{Fe}]$ ) as contours in the $[\mathrm{O} / \mathrm{Fe}]-[\mathrm{Fe} / \mathrm{H}]$ plane. In particular, the contours connect regions of the plane with the same mass fraction of stars. The dotted-dashed line is the average trend (which reflects the mean composition of the gas), while the symbols are a compilation of observations (see Ballero et al. 2007). We consider the stars born in different points of the grid, which may have undergone different chemical evolution histories. This explains why the lowest probability contour (i.e. the outermost) is quite broad. In the single degenerate scenario, the minimum timescale for having a SNIa is $30 \mathrm{Myr}$, therefore we can have stars forming in a cell in which $[\mathrm{O} / \mathrm{Fe}]<1$ even at $[\mathrm{Fe} / \mathrm{H}]=-1.5$, but this is very unlikely. In fact, remarkably, the majority of the observed data points lie within the highest probability contour. In practice our model can explain not only the average trend, but also the intrinsic scatter. The minimum time is in general different for the timescale for SNIa to be effective which is tipically inferred from the position of the knee in the $[\mathrm{O} / \mathrm{Fe}]$ vs $[\mathrm{Fe} / \mathrm{H}]$ curve (dashed line in fig. 1, right panel).

Our fiducial model features a Salpeter IMF, which successfully reproduces the properties of massive spheroids. In fig. 2 (upper-left panel) we compare the stellar metallicity distribution predicted by such a model to two observed K-giants distribution. In the other three panels of fig. 2 we test other possible IMFs, mutivated by either observations or theoretical efforts. In agreement with Matteucci \& Brocato (1990) and Ballero et al. (2007), we find that a Kroupa (2001) IMF poorly fits the observations. On the other hand, an IMF with $\mathrm{x}=0.33$ for $\mathrm{m} \leqslant 1 M_{\odot}$ and $\mathrm{x}=1$ otherwise represents another viable solution. If the exponent $\mathrm{x}=0.33$ is kept constant over the whole mass range predicts too a narrow and peaked stellar metallicity distribution. Moreover the typical $[\mathrm{O} / \mathrm{Fe}]$ of the stars created with this last choice will be too high with respect to the observations. Given the longer infall timescale, the differences between the IMFs are less dramatic than those shown by Ballero et al. (2007). 

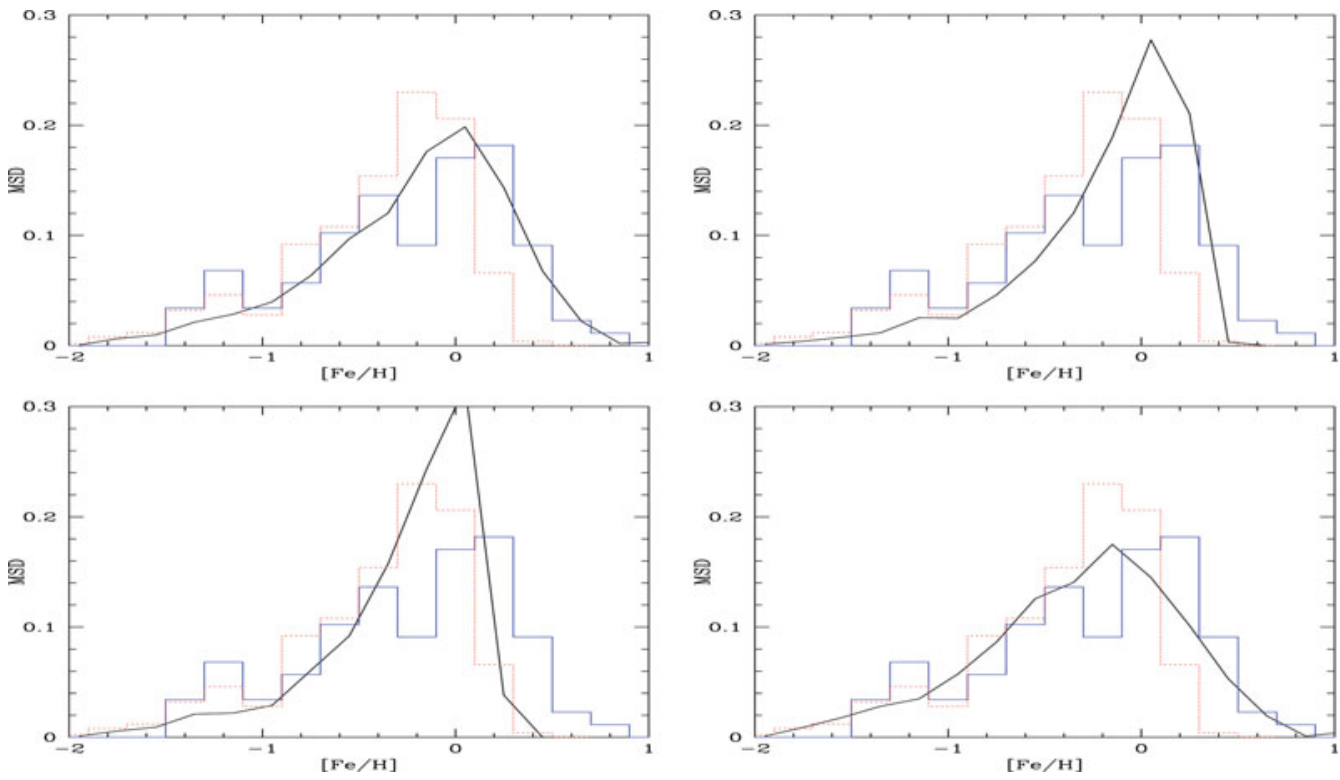

Figure 2. Metallicity distribution function for the stars in the MW bulge. The histograms are the observed K-giants distributions by Fulbright et al. (2006, solid) and Zoccali et al. (2003, dotted) compared to our fiducial model with different IMFs. Upper-left: Salpeter (1955) over the whole mass range. Upper-right: $\mathrm{x}=0.33$ for $\mathrm{m} \leqslant 1 M_{\odot}, \mathrm{x}=1$ otherwise. Lower-left: $\mathrm{x}=0.33$ over the whole mass range. Lower-right: Kroupa (2001) IMF.

Preliminary results shown by Lecureur et al. (this book) seem to suggest a radial variation of stellar metallicity distribution in qualitative agreement with PMC and PDM finding. This topic will be investigated in the future. Finally, we find that the bulge formation in fast in agreement with Matteucci \& Brocato (1990). Star formation stops due to gas consumption rather than to galactic winds (Rich 1988; Ballero et al. 2007).

\section{References}

Annibali, F., Bressan, A., Rampazzo, R., Zeilinger, W. W., \& Danese, L. 2007, A \& A, 463, 455

Ballero, S. K., Matteucci, F., Origlia, L., \& Rich, R. M., 2007, A \& A, 467, 123

Fulbright, J. P., McWilliam, A. \& Rich, M. 2006, ApJ, 636, 821

Goudfrooij, P., Gorgas, J., Jablonka, P., 1999 Ap \& SS, 269, 109

Jablonka, P., Martin, P., \& Arimoto, N. 1996, AL, 112, 1415

Jablonka, P., Gorgas, J. \& Goudfroij, P. 2007, A \& A, arXiv:0707.0561

Kroupa, P. 2001, MNRAS, 332, 231

Matteucci, F. \& Brocato, E. 1990, ApJ, 365, 539

Mehlert, D., Thomas, D., Saglia, R. P., Bender, R., \& Wegner, G. 2003, A\&A, 407, 423

Ogando, R. L. C., Maia, M. A. G., Chiappini, C., Pellegrini, P. S., Schiavon, R. P., \& da Costa, L. N. 2005 ApJ, 632, 61

Pipino, A., D'Ercole, A., \& Matteucci, F. 2007, arXiv:0706.2932

Pipino, A., Matteucci, F., \& Chiappini, C. 2006 ApJ, 638, 739

Proctor, R. N., Sansom, A. E., \& Reid, I. N. 2000, MNRAS, 311, 37

Rich, M. 1998, AJ, 95, 828

Sanchez-Blazquez, P., Forbes, D. A., Strader, J., Brodie, J., \& Proctor, R. 2007, astro$\mathrm{ph} / 0702572$

Thomas, D., \& Davies, R., 2006, MNRAS, 366, 510

Zoccali, M., Renzini, A., Ortolani, S. et al. 2003, A \& A, 399, 931 